bilirubin, $10 \mu \mathrm{mol} / \mathrm{l} ; \gamma \mathrm{GT}, 270 \mathrm{IU} / \mathrm{l})$. The polyneuropathy was relatively quiescent. A second liver biopsy specimen demonstrated a reduction in giant cell transformation, but inflammation and fibrosis, not amounting to cirrhosis, were still present.

\section{Discussion}

Giant cells are present frequently in neonatal liver disease, but are rarely found after infancy. Post-infantile giant cell hepatitis refers to hepatitis associated with extensive giant cell change in the adult liver. It is a purely descriptive term, encompassing a heterologous group of disorders in terms of clinical, serological and histological features. ${ }^{1}$

Aetiological factors implicated include drugs (methotrexate, 6-mercaptopurine and clonmetacin), viruses (hepatitis A, B and C, Epstein-Barr virus and paramyxoviruses) and autoimmune chronic hepatitis. ${ }^{1}$ Devaney et $a l^{2}$ found evidence of autoimmune disease (positive antinuclear antibody or direct Coombs reaction and anaemia) in $40 \%$ of patients.

About $8-23 \%$ of patients with SLE develop liver disease, which is usually of modest clinical relevance and occurs in patients with a higher frequency of positive DNA antibody of double stranded type. ${ }^{3}$ Liver biopsy diagnoses have included granulomatous hepatitis, cirrhosis, chronic active hepatitis, chronic persistent hepatitis, and steatosis. ${ }^{4}$

Giant cell hepatitis has been reported in association with neonatal SLE ${ }^{5}$ and, more recently, with SLE in an adult. ${ }^{6}$ The first two cases of giant cell hepatitis in association with neurological features have also been published recently. ${ }^{1}$ The current case shares similarities in that the patient presented with neurological features and abnormal liver function tests were detected only on routine screening. This suggests that when extensive giant cell transformation is noted on liver biopsy, particularly when neuropathy is also a feature, the possibility of an association with SLE should be considered.

We thank Drs RM Bernstein, JM Braganza and W Schady for permission to report this case.

1 Johnson SJ, Mathew J, MacSween RMN, Bennett MK, Burt ADon SJ, Mathew J, MacSween RMN, Bennett MK, Burt AD. Adult giant cell hepatitis: Histological and im-

2 Devaney K, Goodman ZD, Ishak KG. Post infantile giant cell transformation in hepatitis. Hepatology 1992;16:327-33. 3 Caramaschi P, Biasi D, Botto M, Carletto A, Manzo T Bambara LM. Interessamento epatico nel lupus eritematoso sistemico. Recenti Prog Med 1993;84:673-8.

4 Runyon BA, LaBrecque DR, Anuras S. The spectrum of liver disease in systemic lupus erythematosus. Report of 3 histolorically-proved cases and review of the literature. $A m$ histologically-proved cas

5 Evans $N$, Gaskin $\mathrm{K}$. Liver disease in association with neonatal lupus erythematosus. f Paediatr Child Health 1993;29:478-80. 6 Dohmen K, Ohtsuka S, Nake $\mathrm{Y}$, Asayama R, et al. Post-infantile giant cell hepatitis in an elderly female patient with systemic lupus erythematosus. f Gastroenterol 1994;29:362-8.

\title{
Chaetomium pneumonia in patient with acute myeloid leukaemia
}

\author{
T Yeghen, L Fenelon, C K Campbell, D W Warnock, A V Hoffbrand, H G Prentice, \\ C C Kibbler
}

Department of Microbiology, Royal Free Hospital, Pond Street, Hampstead, London NW3 2QG

$T$ Yeghen

C C Kibbler

Department of

Haematology

A V Hoffbrand

H G Prentice

Mycology Reference Laboratory, Myrtle Road, Kingsdown,

Bristol BS2 8EL

C K Campbell

D W Warnock

Department of Microbiology,

St Vincent's Hospital, Elm Park, Dublin 4, Ireland

L Fenelon

Correspondence to:

Dr T Yeghen.

Accepted for publication

18 October 1995

\begin{abstract}
A patient with relapsed refractory acute myeloid leukaemia developed typical fungal lung lesions despite intravenous amphotericin B prophylaxis. Chaetomium globosum was cultured from the resected right lower lobe. Histology showed branching hyphae negative for common Aspergillus species by immunohistochemical staining. Previous reports of invasive disease caused by Chaetomium and some applications of immunohistochemical staining for Aspergillus are discussed. (f Clin Pathol 1996;49:184-186)
\end{abstract}

Keywords: Chaetomium globosum, pneumonia, immunohistochemical staining, Aspergillus.

Patients with haematological malignancies are susceptible to invasive fungal infections. Although the great majority of such infections are caused by Aspergillus or Candida species, various fungi of low pathogenicity have oc- casionally been reported to cause invasive disease in these immunosuppressed patients.

Because invasive fungal infections are common and devastating complications of cytotoxic therapy in this group of patients, treatment with intravenous amphotericin B is usually introduced within 96 hours of onset of a fever resistant to broad spectrum antibiotics and for which no cause has been determined, or as soon as lesions raising the suspicion of invasive fungal infection (IFI) appear. For those patients undergoing surgical resections as part of the treatment of mycotic lung sequestra (MLS), systemic amphotericin B may already have been given for several days before the operation and, in our institution, fungi are frequently not isolated from the operative sample, so that the only evidence of IFI is the presence of fungal elements on tissue sections.

These hyphae tend to be identified as those of Aspergillus species by default even when not entirely typical. Under such circumstances there is no routine method for proving that the 


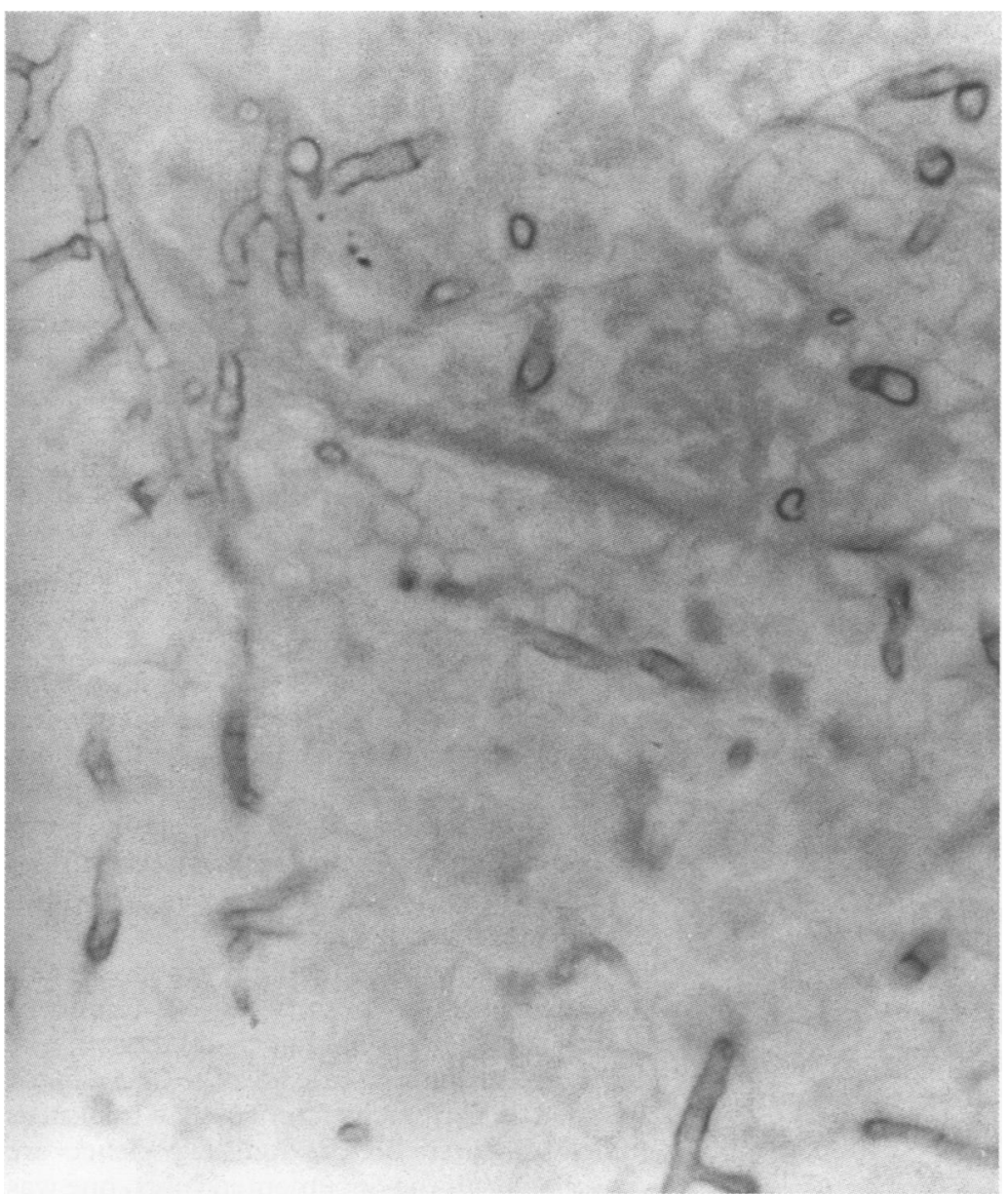

Figure 1 Histological section of lung biopsy specimen, showing branched septate hyphae. (Grocott's silver stain, $\times 1200)$.

hyphae really are Aspergillus. Recently, a specific monoclonal antibody directed against the common species of $A$ spergillus has been developed. ${ }^{1}$ The sensitivity of the antibody in the published

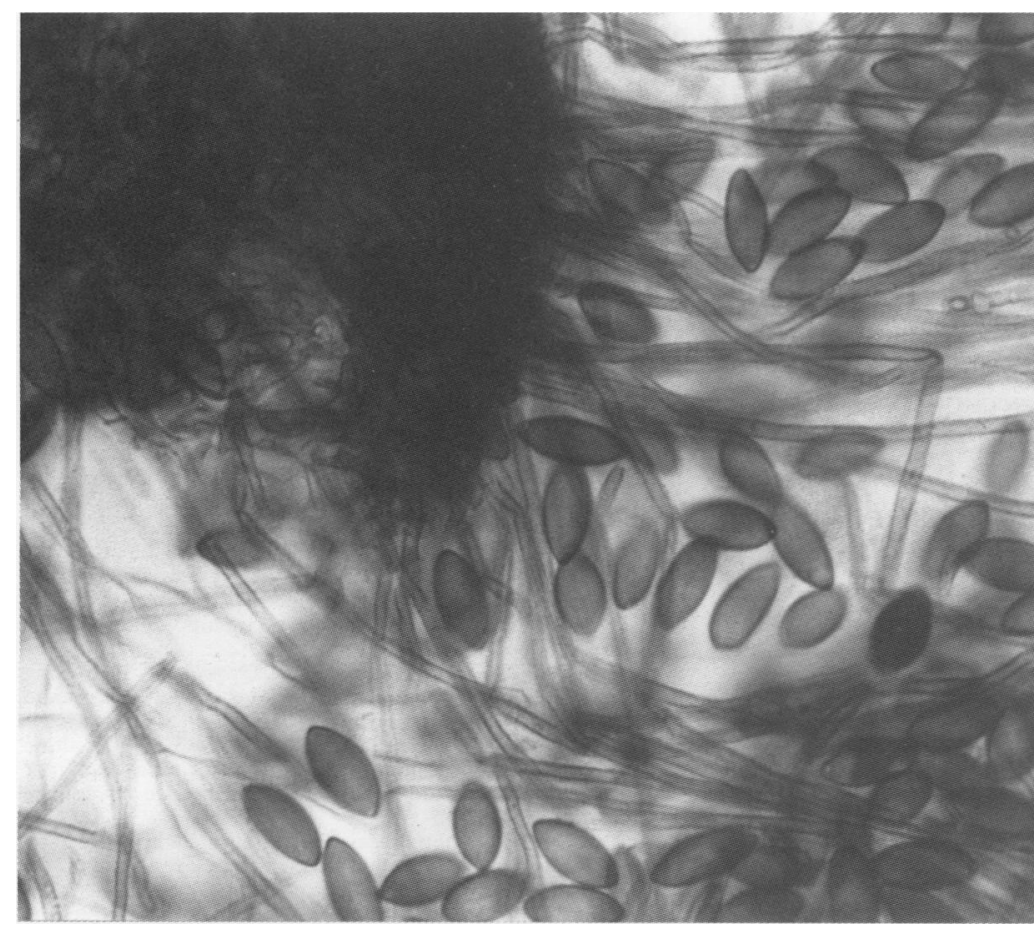

Figure 2 Culture of the isolated strain of Chaetomium globosum, showing ruptured ascocarp wall and ascospores $(\times 1200)$. study is $88 \cdot 8 \%$, and its specificity $100 \%$. This antibody may be helpful in clarifying the epidemiology of culture negative, histology positive IFI.

Chaetomium species are rarely implicated in human pathology, but are a well recognised, albeit uncommon, cause of onychomycosis in people with normal immunity. Chaetomium has been reported as a confirmed or suspected pathogen in the immunocompromised host on four occasions only. ${ }^{2-5}$ Empyema was described once $^{4}$ in association with Chaetomium. In another case Chaetomium was grown from the bronchoalveolar lavage specimen of a patient whose pulmonary radiological changes probably preceded her admission to hospital, and who was nursed in a room where three patients colonised with the organism had been housed before. The air filter system was subsequently found to be contaminated with Chaetomium and it is likely that the isolate in this patient represented colonisation rather than infection. ${ }^{3}$ We report what we believe is the first case of Chaetomium pneumonia in a patient with acute myeloblastic leukaemia (AML) and highlight potential difficulty in identifying the causal organism in IFI.

\section{Case report}

A 19 year old man from Israel was diagnosed with AML FAB type M4 Eo in March 1993. Cytogenetic studies showed inversion 16 and 7q-. He received two courses of chemotherapy in his own country and achieved remission. The second course was apparently complicated by "pulmonary infiltrates suggestive of invasive aspergillosis". He responded to intravenous amphotericin B although no fungi were isolated from his sputum and no bronchoalveolar lavage was performed. He received one further course of consolidation chemotherapy but suffered a leukaemic relapse one month later. Re-induction was not successful, and he was transferred to our institution for further treatment.

On admission, a computed tomography (CT) scan of his chest was normal and a CT scan of his sinuses showed no evidence of aspergillosis. His bone marrow was heavily infiltrated with leukaemic cells and he had no circulating neutrophils. He received amphotericin B $1 \mathrm{mg} / \mathrm{kg}$ on alternate days as secondary antifungal prophylaxis and a course of modified timed sequential chemotherapy ${ }^{67}$ was given.

After nine days on amphotericin $B$, the patient's renal function deteriorated and prophylaxis was changed to liposomal amphotericin $B 1 \mathrm{mg} / \mathrm{kg}$ daily.

On day 24 of re-induction chemotherapy, the patient developed right sided pleuritic chest pain and fever. He remained totally neutropenic. Chest $x$ ray and CT scan showed cavitating lesions in the right lower lobe. The dose of liposomal amphotericin $\mathrm{B}$ was increased to $5 \mathrm{mg} / \mathrm{kg}$ daily and a right lower lobectomy was performed. Histology revealed branching hyphae invading blood vessels (fig 1). The hyphae were fragmented and were not present in sufficient numbers to permit further characterisation. A filamentous fungus, off-white in 
colour, was grown on one of three Sabouraud plates (the $37^{\circ} \mathrm{C}$ plate) from the lobectomy specimen pool. Septate hyphae were seen, but no other characteristics were noted.

The fungus was submitted to the Mycology Reference Laboratory where it was subcultured onto slopes of Sabouraud's glucose agar, potato sucrose agar and Borelli's lactrimel agar, and incubated at $30^{\circ} \mathrm{C}$ in the dark. After one week, the off-white mould had developed a central dark pigment, especially on the lactrimel agar, but no sporing structures were produced after one month and the cultures were allowed to continue to grow at room temperature in natural light. After growth in these conditions for a further six weeks, the culture on the lactrimel plate contained mature perithecia of $C$ globosum (fig 2). An attempt to repeat subcultures from the submitted culture which had been stored at $4^{\circ} \mathrm{C}$ for nearly three months resulted in no growth. However, a subculture stored at the Royal Free Hospital and submitted to the Mycology Reference Laboratory was found to be C globosum.

We were concerned that the $C$ globosum might be a contaminant and that an Aspergillus species, which had not been successfully isolated, was the real cause of the infection. In order to investigate this, immunohistochemical staining of sections of the affected lung was performed, using a monoclonal antibody directed against the commonest species of Aspergillus found in human disease: $A$ fumigatus, $A$ flavus, and $A$ niger. ${ }^{1}$ Aspergillus hyphae were not seen on examination of these lung sections, suggesting that $C$ globosum was indeed the cause of the infection.

The patient did not achieve remission and salvage chemotherapy was attempted with high dose melphalan with a view to proceeding to a volunteer unrelated donor transplant.

He developed new cavitating lesions in the right lung seven weeks after the initial operation in spite of continuous treatment with high doses of liposomal amphotericin B ( $5 \mathrm{mg} / \mathrm{kg})$. He had remained profoundly neutropenic throughout. A right pneumonectomy was performed in midFebruary 1994. Two weeks following surgery, after a good initial recovery, he suffered a respiratory arrest and died in spite of respiratory support. No fungi were isolated from the pneumonectomy sample, but branching hyphae were again seen in tissue sections. A necropsy was not performed.

The patient had died before the Chaetomium was characterised, and the results of the immunostaining became available.

The culture of $C$ globosum was added to the UK National Collection of Pathogenic Fungi as NCPF 7115.

\section{Discussion}

Speciation of fungi involved in invasive infection in neutropenic patients is not possible when such an infection is diagnosed by histological examination with no confirmatory culture results. Although Aspergillus is said to have characteristic appearances in tissue sections (dichotomous branching at $45^{\circ}$ ), a definite diagnosis is not possible if few hyphae are present, or the invaded part of the tissue small. Fusariosis may give similar appearances, although this infection is extremely rare in our institution

A precise diagnosis is not always considered necessary for therapeutic purposes, as amphotericin $B$ is the drug of choice for most infections with filamentous fungi. Fusarium species and Pseudallescheria boydii respond poorly to this drug, however, and confirmed infection with these organisms warrants a change of treatment to an azole antifungal. Exclusion of an Aspergillus infection may thus be useful for therapeutic purposes, given the different treatment required for these alternative pathogens.

In our case, the fungus that was isolated is a rare pathogen and a frequent contaminant. It might have been dismissed as such without the immunopathological study. Although proving that a common species of Aspergillus was not responsible for the patient's infection does not prove that the Chaetomium was the pathogen, it does make it more likely.

There were no treatment implications in this particular instance, both because a fulminant clinical course preceded full characterisation of the organism, and because invasive Chaetomium infection is extremely rare, so that treatment guidelines based on previous experience are unavailable. Of the documented cases, one was not treated with systemic antifungals, ${ }^{2}$ one was treated with intravenous amphotericin B with initial success, ${ }^{4}$ and the third-the only case where in vitro sensitivity tests were carried out - was resistant to amphotericin B but sensitive to ketoconazole, which was given to the patient with good clinical, but poor mycological response ${ }^{5}$; isolation of Chaetomium ceased only after removal of the peritoneal dialysis catheter.

In conclusion, immunostaining was helpful on this occasion in excluding Aspergillus infection and ascribing pathogenicity to $C$ globosum in a case of fungal pneumonia. The development of immunostaining techniques capable of identifying a variety of different fungi would be a considerable aid in the investigation of invasive fungal infections.

1 Fenelon LE, Hamilton AJ, Figueroa J, Allen MH, Hay RJ. Immunohistochemical diagnosis of aspergillosis using monoclonal antibodies. In: editors. Proceedings of the 2nd Western Pacific Conference on Infectious Diseases and Chemotherapy; 1990 Dec 11-14; Tomtien-Pattya, Thailand. Western Pacific Society of Chemotherapy, 1990:352.

2 Anandi V, John TJ, Walter A, Shastry JCM, Lalitha MK, Padhye AA, et al. Cerebral phaeohyphomycosis caused by Chaetomium globosum in

3 Woods GL, Davis JC, Vaughan WP. Failure of the sterile air flow component of a protected environment detected by demonstration of Chaetomium species colonization of four consecutive immunosuppressed occupants. Infect Control Hosp Epidemiol 1988;9:451-6.

4 Hoppin EC, McCoy EL, Rinaldi MG. Opportunistic mycotic infection caused by Chaetomium in a patient with acute leukaemia. Cancer 1983;52:555-6.

5 Barthez JP, Pierre D, De Bièvre C, Arbeille M. Péritonite à Chaetomium globosum chez un insufisant rénal traité par D.P.C.A. Bulletin de la Societé Française de Mycologie Médicale 1984;13:205-8.

6 Burke PJ, Karp E. Long remissions in adults with leukaemia treated with two courses of timed drugs. Proceedings of the American Society of Clinical Oncology 1987;6:600.

7 Vaughan WP, Karp JE, Burke PJ. Two cycle timed sequential Vaughan WP, Karp JE, Burke PJ. Two cycle timed sequential Blood 1984;74:975-80. 